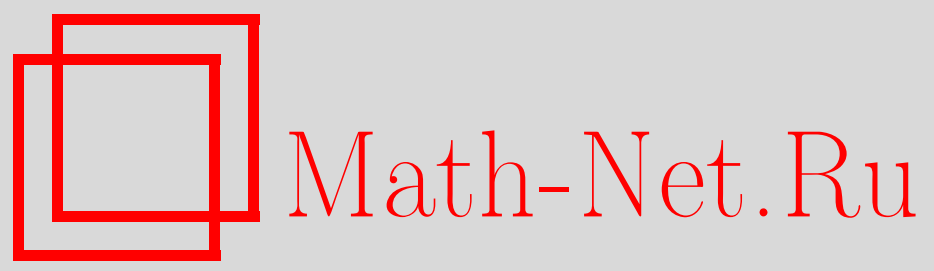

О. В. Камловский, Неабсолютные оценки для неполных тригонометрических сумм от линейных рекуррент и их приложения, Матем. вопр. криптогр., 2014, том 5, выпуск 3, 17-34

DOI: https://doi.org/10.4213/mvk127

Использование Общероссийского математического портала Math-Net.Ru подразумевает, что вы прочитали и согласны с пользовательским соглашением http://www.mathnet.ru/rus/agreement

Параметры загрузки:

IP : 3.85 .73 .92

26 апреля 2023 г., $17: 36: 20$ 
МАТЕМАТИЧЕСКИЕ ВОПРОСЫ КРИПТОГРАФИИ

2014 Т. 5 № 3 C. $17-34$

УДК 511.216+519.113.6

\title{
Неабсолютные оценки для неполных тригонометрических сумм от линейных рекуррент и их приложения
}

\author{
О. В. Камловский
}

ООО "Центр сертификационных исследований г. Москва

Получено 22.IV.2013

Устанавливаются верхние оценки для некоторых классов неполных тригонометрических сумм над кольцами Галуа. Указываются приложения данных результатов к исследованию коэффициентов кросскорреляции и частот элементов в линейных рекуррентных последовательностях.

Ключевые слова: линейные рекуррентные последовательности, неполные тригонометрические суммы, кольца Галуа, коэффициенты кросскорреляции

Nonabsolute bounds for incomplete exponential sums of elements of linear recurrent sequences and their applications

O. V. Kamlovsky

LLC "Sertification Research Center", Moscow

Abstract. We establish several upper bounds for some classes of incomplete exponential sums over Galois rings. We apply these results to crosscorrelation coefficients and distribution properties of linear recurrent sequences.

Keywords: linear recurrent sequences, incomplete exponential sums, Galois rings, cross-correlation coefficients

Citation: Mathematical Aspects of Cryptography, 2014, vol. 5, no. 3, pp. 17-34 (Russian)

(C) 2014 О. В. Камловский 


\section{1. Введение}

Ряд задач, связанных с распределением элементов на отрезках псевдослучайной последовательности $u$ над конечным коммутативным кольцом $R$, сводится к исследованию так называемых неполных тригонометрических сумм, т. е. таких сумм в которых суммирование осуществляется по знакам последовательности $u$, расположенным на отрезке длины $l$, меньшей ее периода $T(u)$. Среди таких сумм наиболее простыми и часто используемыми являются суммы вида

$$
\sum_{i=0}^{l-1} \chi(u(i)),
$$

где $\chi$ - аддитивный характер кольца $R$.

В случае, когда $R=G F(q)$ - поле из $q$ элементов, для модуля таких сумм были получены абсолютные (не зависящие от $l$ ) оценки сверху, которые оказались асимптотически неулучшаемыми (см. [1]-[5]). Первая неабсолютная оценка для тригонометрических сумм вида (1) в случае, когда $q$ - простое число, была получена в $[6$, теорема 1]. В этой же работе было показано, что при некоторых значениях $l$ она точнее известных неабсолютных оценок. Другой метод получения неабсолютных оценок для сумм вида (1) был предложен в [7] и развивался в большом количестве работ (см., например, обзор литературы в [8]). Он оказался эффективным для некоторых классов последовательностей $u$, удовлетворяющих условию $T(u)=|R|$. Вопрос об эффективности этого метода для последовательностей, у которых $T(u)$ значительно больше величины $|R|$, остается пока открытым. В данной работе исследуется именно эта ситуация. Рассматривается один класс неполных тригонометрических сумм над кольцом $R$, включающий в себя суммы вида (1). Для этого класса предлагается общий метод получения неабсолютных оценок, который является развитием идей из [6], [9, § 5], а также статей автора [10]-[12].

В настоящей статье нетривиальные оценки для исследуемого класса тригонометрических сумм устанавливаются в случае, когда $R$ - кольцо Галуа (в частности, конечное поле). Полученные результаты применяются для оценок параметров линейных рекуррент: коэффициентов кросскорреляции и чисел появлений элементов кольца $R$ на отрезках. Показывается, что в некоторых случаях эти результаты уточняют ранее известные абсолютные оценки исследуемых параметров. 


\section{2. Общий подход к исследованию неполных сумм}

Пусть $R$ - конечное коммутативное кольцо, $\chi$ - нетривиальный характер группы $(R,+), u=(u(i))_{i=0}^{\infty}$ - чисто периодическая последовательность элементов кольца $R$, а $H$ - некоторое непустое подмножество кольца $R$. Для каждого элемента $z \in R$ и натурального числа $l$ рассмотрим тригонометрическую сумму

$$
\tau_{l}(z, u, H)=\sum_{a \in H} \chi(-a z) \sum_{i=0}^{l-1} \chi(a u(i)) .
$$

Предложим подход, позволяющий оценить сверху модуль рассматриваемой суммы.

Обозначим через $P(u)$ множество всех сдвигов последовательности $u$, т. е. множество всех последовательностей вида $x^{0} u, x^{1} u, x^{2} u, \ldots$, где $x^{j} u=(u(j), u(j+1), \ldots), j \in \mathbb{N}_{0}$. Если $t=T(u)$ - период последовательности $u$, то

$$
P(u)=\left\{x^{0} u, x^{1} u, x^{2} u, \ldots, x^{t-1} u\right\} .
$$

Заметим, что каждая последовательность $v \in P(u)$ является чисто периодической последовательностью периода $t$. Обозначим через $s=s(H)$ такое натуральное число, что для всех $j \in\{0,1, \ldots, t-1\}$ и всех $v \in P(u)$ имеют место неравенства

$$
\left|\tau_{l}(z, v, H)-\tau_{l}\left(z, x^{j} v, H\right)\right| \leq s j, \quad\left|\tau_{l}(z, v, H)-\tau_{l}\left(z, x^{t-j} v, H\right)\right| \leq s j .
$$

Утверждение 1. Существует натуральное число s, при котором имеют место неравенства (3), причем его можно подобрать так, что $s \leq 2|H|$.

Доказательство. Векторы $(v(0), v(1), \ldots, v(l-1))$ и $(v(j), v(j+$ $1), \ldots, v(j+l-1))$ при $j<l$ имеют по крайней мере $l-j$ общих элементов $v(j), \ldots, v(l-1)$. Тогда

$$
\begin{aligned}
\tau_{l}(z, v, H)- & \tau_{l}\left(z, x^{j} v, H\right)=\sum_{a \in H} \chi(-a z)\left(\sum_{i=0}^{j-1} \chi(a v(i))-\sum_{k=l}^{l+j-1} \chi(a v(k))\right)= \\
& =\sum_{a \in H}\left(\sum_{i=0}^{j-1} \chi(a(v(i)-z))-\sum_{k=l}^{l+j-1} \chi(a(v(k)-z))\right) .
\end{aligned}
$$

Таким образом величина $\tau_{l}(z, v, H)-\tau_{l}\left(z, x^{j} v, H\right)$ равна сумме не более $2|H| j$ значений характера $\chi$, а значит,

$$
\left|\tau_{l}(z, v, H)-\tau_{l}\left(z, x^{j} v, H\right)\right| \leq 2|H| j .
$$


Очевидно, это неравенство выполнено и при $j \geq l$. Аналогично в силу того, что векторы $(v(0), v(1), \ldots, v(l-1))$ и $(v(t-j), v(t-j+1), \ldots, v(t-$ $j+l-1))$ при $j<l$ имеют по крайней мере $l-j$ общих элементов $v(0)$, $\ldots, v(l-j-1)$, получим

$$
\left|\tau_{l}(z, v, H)-\tau_{l}\left(z, x^{t-j} v, H\right)\right| \leq 2|H| j .
$$

Это неравенство выполнено и при $j \geq l$.

В дальнейшем понадобится следующее вспомогательное утверждение.

Лемма 1 ([10]). Для всех неотрииательных действительных чисел $x$ u всех натуральных чисел $s$

$$
x^{2}+2 \sum_{j=1}^{[x / s]}(x-s j)^{2} \geq \frac{2 x^{3}}{3 s},
$$

где $[x / s]$ - челая часть числа $x / s$.

Теорема 1. Пусть $R$ - конечное коммутативное кольцо, $H$ - непустое подмножество в $R, u$ - чисто периодическая последовательность над кольиом $R$. Тогда для всех $z \in R$ и всех таких натуральных l, чmo

$$
l<\frac{T(u) s}{2|H|},
$$

выполнено неравенство

$$
\left|\tau_{l}(z, u, H)\right| \leq\left(\frac{3 s}{2} \sum_{v \in P(u)}\left|\tau_{l}(z, v, H)\right|^{2}\right)^{\frac{1}{3}} .
$$

Доказательство. Выберем последовательность $v_{0} \in P(u)$, имеющую максимальное величины величины $\left|\tau_{l}(z, v, H)\right|$ среди всех ЛРП $v \in P(u)$. Пусть $\tau=\left|\tau_{l}\left(z, v_{0}, H\right)\right|$. Докажем, что

$$
\tau \leq\left(\frac{3 s}{2} \sum_{v \in P(u)}\left|\tau_{l}(z, v, H)\right|^{2}\right)^{\frac{1}{3}} .
$$

Отсюда будет следовать справедливость доказываемого неравенства. Пусть $t=T(u)$. Для всех $j \in\{0,1, \ldots, t-1\}$ положим

$$
v_{j}=x^{j} v_{0}, \quad v_{-j}=x^{t-j} v_{0} .
$$


Из определения величины $\tau_{l}(z, v, H)$ следует, что $\tau \leq|H| l$, а значит $[\tau / s] \leq \tau / s \leq|H| l / s<t / 2$. Это гарантирует, что все последовательности

$$
v_{-\left[\frac{\tau}{s}\right]}, \ldots, v_{0}, v_{1}, \ldots, v_{\left[\frac{\tau}{s}\right]}
$$

являются попарно различными. Согласно неравенствам (3)

$$
\left|\tau_{l}\left(z, v_{j}, H\right)\right| \geq \tau-s j, \quad\left|\tau_{l}\left(z, v_{-j}, H\right)\right| \geq \tau-s j, \quad j=0,1, \ldots, t-1
$$

а тогда с использованием леммы 1 получим

$$
\sum_{v \in P(u)}\left|\tau_{l}(z, v, H)\right|^{2}=\sum_{v \in P\left(v_{0}\right)}\left|\tau_{l}(z, v, H)\right|^{2} \geq \tau^{2}+2 \sum_{j=1}^{[\tau / s]}(\tau-s j)^{2} \geq \frac{2 \tau^{3}}{3 s} .
$$

Таким образом неравенство (4) доказано.

Теорема 1 сводит исследование неполной суммы $\tau_{l}(z, u, H)$ к исследованию суммы

$$
\sum_{v \in P(u)}\left|\tau_{l}(z, v, H)\right|^{2}
$$

и устанавливает альтернативный способ по сравнению с классическим переходом от исследования неполных тригонометрических сумм к изучению некоторых полных тригонометрических сумм (см. [13, теорема 2]).

\section{3. Оценки тригонометрических сумм}

Рассмотрим случай, когда $R=P=G F(q), u$ - ненулевая ЛРП над полем $P$ с характеристическим многочленом $F(x) \in P[x]$. Согласно теореме 1 для оценки суммы $\tau_{l}(z, u, H)$, определенной равенством (2), достаточно получить оценку сверху суммы, заданной равенством (5). В качестве такой оценки рассмотрим величину

$$
\sum_{v \in L_{P}(F) \backslash\{(0)\}}\left|\tau_{l}(z, v, H)\right|^{2},
$$

где $L_{P}(F)$ - множество всех ЛРП над полем $P$ с характеристическим многочленом $F(x)$, а $(0)$ - нулевая последовательность.

Утверждение 2. Пусть $F(x)$ - неприводимый многочлен степени $m$ над полем $P=G F(q), F(x) \neq x, t=T(F)$ - период многочлена $F(x)$, 
$H-$ nодгруппа мультипликативной группы $P^{*}$. Тогда для всех $z \in P$ и всех таких натуральных $l$, что $l \leq t /(t,|H|)$, справедливо равенство

$$
\sum_{v \in L_{P}(F) \backslash\{(0)\}}\left|\tau_{l}(z, v, H)\right|^{2}=|H| q^{m} l-\varkappa(z, H) l^{2},
$$

где

$$
\varkappa(z, H)=\left|\sum_{a \in H} \chi(a z)\right|^{2} .
$$

Доказательство. Введем обозначение

$$
S_{l}(z, F, H)=\sum_{v \in L_{P}(F)}\left|\tau_{l}(z, v, H)\right|^{2} .
$$

Из равенства $(2)$ и соотношения $\chi(-a z)=\overline{\chi(a z)}$, где черта означает комплексное сопряжение, имеем

$$
\left|\tau_{l}(z,(0), H)\right|^{2}=\left|\sum_{a \in H} \chi(-a z)\right|^{2} l^{2}=\varkappa(z, H) l^{2} .
$$

Поэтому достаточно показать, что

$$
S_{l}(z, F, H)=|H| q^{m} l .
$$

Представим элементы последовательности $v$ следующим образом:

$$
v(i)=\operatorname{tr}_{P}^{Q}\left(b \alpha^{i}\right), \quad i \geq 0,
$$

где $Q=G F\left(q^{m}\right), \operatorname{tr}_{P}^{Q}-$ функция следа из поля $Q$ в поле $P, \alpha-$ корень многочлена $F(x)$ в поле $Q, b$ - однозначно определенный элемент поля $Q[2]$. Пусть $\chi^{\prime}-$ поднятие характера $\chi$ до поля $Q$, т. е. $\chi^{\prime}(x)=\chi\left(\operatorname{tr}_{P}^{Q}(x)\right)$ для всех $x \in Q$, тогда согласно равенству (2)

$$
\begin{gathered}
S_{l}(z, F, H)=\sum_{b \in Q}\left(\sum_{c \in H} \chi(-c z) \sum_{i=0}^{l-1} \chi^{\prime}\left(c b \alpha^{i}\right)\right)\left(\overline{\sum_{d \in H} \chi(-d z) \sum_{j=0}^{l-1} \chi^{\prime}\left(d b \alpha^{j}\right)}\right)= \\
=\sum_{b \in Q} \sum_{c, d \in H} \chi((d-c) z) \sum_{i, j=0}^{l-1} \chi^{\prime}\left(b\left(c \alpha^{i}-d \alpha^{j}\right)\right) .
\end{gathered}
$$


Изменив порядок суммирования, получим

$$
S_{l}(z, F, H)=\sum_{c, d \in H} \chi((d-c) z) \sum_{i, j=0}^{l-1} \sum_{b \in Q} \chi^{\prime}\left(b\left(c \alpha^{i}-d \alpha^{j}\right)\right) .
$$

С использованием соотношения ортогональности для характеров (см., например, [2, равенство (5.8)]) будем иметь

$$
\sum_{b \in Q} \chi^{\prime}\left(b\left(c \alpha^{i}-d \alpha^{j}\right)\right)= \begin{cases}q^{m}, & \text { если } c \alpha^{i}=d \alpha^{j} \\ 0, & \text { если } c \alpha^{i} \neq d \alpha^{j}\end{cases}
$$

Равенство $c \alpha^{i}=d \alpha^{j}$ приводит к соотношению $c d^{-1}=\alpha^{j-i}$, т. е. $\alpha^{j-i}$ будет являться элементом группы $H$. Это приведет к соотношению $\alpha^{(j-i)|H|}=$ $e$, где $e$ - единица поля $P$, что возможно только если $t /(t,|H|)$ делит $j-i$. Учитывая, что $i, j \in\{0,1, \ldots, l-1\}$ и $l \leq t /(t,|H|)$, получим: равенство $c \alpha^{i}=d \alpha^{j}$ имеет место тогда и только тогда, когда $i=j$ и $c=d$. В итоге из равенства (10) будем иметь $S_{l}(z, F, H)=|H| q^{m} l$.

Отметим, что в общем случае найти точное значение величины $\varkappa(z, H)$, заданной равенством $(8)$, не удается. Однако в частных случаях это можно сделать, например, если $H=\{e\}$, то

$$
\varkappa(z, H)=1, \quad z \in P,
$$

а если $H=P^{*}$, то

$$
\varkappa(z, H)= \begin{cases}1 & \text { при } z \neq 0 \\ (q-1)^{2} & \text { при } z=0\end{cases}
$$

При некоторых $H$ получим оценки для сумм $\tau_{l}(z, u, H)$, определенных равенством (2).

Теорема 2. Пусть $P=G F(q), F(x)$ - неприводимый многочлен степени $m$ над полем $P, F(x) \neq x, H=\{e\}$. Тогда для кажсдой ненулевой ЛРП $u \in L_{P}(F)$, каждого $z \in P$ и каждого такого натурального числа $l$, чmo $l<T(F)$,

$$
\left|\tau_{l}(z, u, H)\right| \leq\left(3\left(q^{m} l-l^{2}\right)\right)^{\frac{1}{3}} .
$$

Доказательство. Согласно утверждению 1 и теореме 1

$$
\left|\tau_{l}(z, u, H)\right|=\left|\sum_{i=0}^{l-1} \chi(u(i))\right| \leq\left(3 \sum_{v \in P(u)}\left|\tau_{l}(z, v,\{e\})\right|^{2}\right)^{\frac{1}{3}}
$$


Остается заметить, что по утверждению 2 и равенству (11)

$$
\sum_{v \in P(u)}\left|\tau_{l}(z, v,\{e\})\right|^{2} \leq S_{l}(z, F,\{e\})=q^{m} l-l^{2} .
$$

Отметим, что ранее теорема 2 была доказана в работе [6, теорема 1] для частного случая, когда $P$ - простое поле.

Следующий результат независимо доказан автором и Биляком И.Б. (см. [14]).

Теорема 3. Пусть выполнены условия теоремы 2 с $H=P^{*}$, а l удовлетворяет дополнительньм условиям $l \leq t /(t, q-1)$ u $l<t q /(2(q-1))$, где $t=T(F)$, тогда

$$
\left|\tau_{l}(z, u, H)\right| \leq\left(\frac{3 q}{2}\left((q-1) q^{m} l-l^{2}\right)\right)^{\frac{1}{3}}
$$

Доказательство. По теореме 1, утверждению 2 и равенству (12) для всех таких $l$, что $l<t s /(2(q-1))$, имеем

$$
\left|\tau_{l}(z, u, H)\right| \leq\left(\frac{3 s}{2}\left((q-1) q^{m} l-\delta(z) l^{2}\right)\right)^{\frac{1}{3}},
$$

где число $s$ определено соотношениями $(3)$, а $\delta(z)-$ равенствами

$$
\delta(z)= \begin{cases}1, & \text { если } z \neq 0 \\ (q-1)^{2}, & \text { если } z=0 .\end{cases}
$$

Остается показать, что при $H=P^{*}$ годится число $s=q$. Пусть $N_{l}(z, u)$ - число появлений элемента z среди элементов $u(0), u(1), \ldots, u(l-1)$. использованием соотношения ортогональности для характеров получим

$$
N_{l}(z, u)=\frac{1}{q} \sum_{a \in P} \chi(a(u(i)-z))=\frac{l}{q}+\frac{\tau_{l}(z, u, H)}{q} .
$$

Tak кak

$$
\tau_{l}\left(z, x^{j} v, H\right)=q N_{l}\left(z, x^{j} v\right)-l, \tau_{l}\left(z, x^{t-j} v, H\right)=q N_{l}\left(z, x^{t-j} v\right)-l
$$

и $\left|N_{l}(z, v)-N_{l}\left(z, x^{j} v\right)\right| \leq j,\left|N_{l}(z, v)-N_{l}\left(z, x^{t-j} v\right)\right| \leq j$, то для всех $j \in\{0,1, \ldots, t-1\}$ и всех $v \in P(u)$ выполнены неравенства

$$
\left|\tau_{l}(z, v, H)-\tau_{l}\left(z, x^{j} v, H\right)\right| \leq q j, \quad\left|\tau_{l}(z, v, H)-\tau_{l}\left(z, x^{t-j} v, H\right)\right| \leq q j .
$$


Пусть $R=G R\left(q^{n}, p^{n}\right)$ - кольцо Галуа характеристики $p^{n}$, состоящее из $q^{n}$ элементов, где $p$ - простое число, а $q=p^{t}$ для некоторого натурального числа $t[15]$. Рассмотрим такой унитарный многочлен $F(x) \in R[x]$, что его образ $\bar{F}(x)$ при действии естественного эпиморфизма колец $R[x] \rightarrow \bar{R}[x]$ неприводим над полем $\bar{R}=R / p R=G F(q)$ и отличен от $x$. Назовем такой многочлен реверсивным многочленом Галуа. Период $T(F)$ такого многочлена равен $p^{\nu} T(\bar{F})=p^{\nu}\left(q^{m}-1\right) / d$, где $0 \leq \nu \leq n-1$, а $d$ - делитель $q^{m}-1[16]$.

Обозначим через $L_{R}(F)$ множество всех ЛРП над кольцом $R$ с характеристическим многочленом $F(x)$. Рассмотрим тригонометрическую сумму $\tau_{l}(z, u, H)$, определенную равенством (2), в котором $u \in L_{R}(F)$, а $\chi$ - аддитивный характер кольца $R$, задаваемый равенством

$$
\chi(x)=\exp \left\{2 \pi i \frac{\operatorname{Tr}_{R_{0}}^{R}(x)}{p^{n}}\right\}, \quad x \in R,
$$

где $i-$ мнимая комплексная единица, $R_{0}=\left\{0, e, 2 e, \ldots,\left(p^{n}-1\right) e\right\}-$ подкольцо, порожденное единицей $e$, a $\operatorname{Tr}_{R_{0}}^{R}-$ функция следа из кольца $R$ в подкольцо $R_{0}$.

Согласно теореме 1 для оценки сверху модуля суммы $\tau_{l}(z, u, H)$ необходимо оценить сверху сумму (5). В отличие от случая конечного поля, для оценки сверху последней суммы не имеет смысла использовать величину

$$
\sum_{v \in L_{R}(F) \backslash\{(0)\}}\left|\tau_{l}(z, v, H)\right|^{2},
$$

так как получатся тривиальные оценки. Однако можно использовать оценку для сумм Вейля над кольцами Галуа, полученную в [17]. С использованием этой идеи оценки суммы (5) были получены в [11] (для случая $H=\{e\})$ и в [12] (в случае, когда $H=R^{*}$ - мультипликативная группа кольца $R$ ). Приведем два следствия из результатов этих работ.

Теорема 4. Пусть $F(x)$ - реверсивный многочлен Галуа степени $m$ над кольиом $R, u$ - ненулевая ЛРП из множества $L_{R}(F), l<T(\bar{F})$, $H=\{e\}$, тогда

$$
\left|\tau_{l}(z, u, H)\right| \leq\left(\frac{3 p^{\nu} l}{d}\left(q^{m}-l+(l-1)\left(d p^{n-1}-1\right) q^{\frac{m}{2}}\right)\right)^{\frac{1}{3}} .
$$

Сравним оценку из теоремы 4 в частном случае, когда $n=1(R=$ $G R(q, p)=G F(q))$ с оценкой из теоремы 2 . Если $d=1$, то рассматриваемые оценки совпадают, а если $d>1$, то оценка из теоремы 2 точнее тогда 
и только тогда, когда $l>q^{m / 2}$. Как будет показано в следующем параграфе, такие значения $l$ являются наиболее важными для приложений, так как при них оценки становятся нетривиальными.

Для ЛРП $u \in L_{R}(F)$ (элемента $z \in R$ ) обозначим через $\|u\|(\|z\|)$ наибольшее из таких чисел $k \in\{0,1, \ldots, n\}$, что $u \in p^{k} L_{R}(F)\left(z \in p^{k} R\right)$.

Теорема 5. Пусть выполнены условия теоремы 4, с $H=R^{*},\|u\|=k$, $f=q^{n-1}(q-1), t=T(\bar{F}),\|z\| \geq k, l \leq t /(t, q-1) u l<t q /(2(q-1))$, mогдa

$$
\left|\tau_{l}(z, u, H)\right| \leq\left(\frac{3 p^{\nu} q^{n} f l}{2 d}\left(q^{m+k}-\delta_{k} l+\left(l f-q^{k}\right)\left(d p^{n-1}-1\right) q^{\frac{m}{2}}\right)\right)^{\frac{1}{3}},
$$

где

$$
\delta_{k}=\left\{\begin{array}{cl}
q^{2(n-1)} / f, & \text { если } k=n-1, \\
0, & \text { если } k<n-1 .
\end{array}\right.
$$

В ситуации, когда $R=G F(q)(n=1)$, оценка из теоремы 5 совпадает с оценкой из теоремы 3 только при $d=1$, а при $d>1$ и всех $l \geq 2$ она является менее точной.

Таким образом, теоремы 4 и 5 являются обобщениями на случай произвольного кольца Галуа $R=G R\left(q^{n}, p^{n}\right)$ теорем 2 и 3 соответственно лишь в классе ЛРП, характеристический многочлен $F(x)$ которых удовлетворяет условию $T(\bar{F})=q^{m}-1$. В случае $T(\bar{F})<q^{m}-1$ оценки из теорем 2 и 3 являются более точными практически для всех интересующих нас значений $l$.

Нами изучены тригонометрические суммы (2) в частном случае, когда $H=\{e\}$ и $H=R^{*}$. Исследование этих сумм при других подмножествах $H \subset R$ пока остается открытой проблемой.

\section{4. Коэффициенты кросс-корреляции}

Пусть $R=G R\left(q^{n}, p^{n}\right), \chi$ - аддитивный характер кольца $R$, определенный равенством (13). Коэффиииент кросс-корреляиии между последовательностями $u$ и $v$ определяется для всех целых неотрицательных чисел $j, l$ равенством

$$
C_{u, v}(j, l)=\sum_{i=0}^{l-1} \chi(u(i)-v(i+j)) .
$$

В случае, когда $u=v$, коэффициент $C_{u, v}(j, l)$ называется коэффиииентом автокорреляиии. Величина $C_{u, v}(j, l)$ характеризует "близость" отрезков $(u(0), \ldots, u(l-1))$ и $(v(j), \ldots, v(j+l-1))$. В случае 
поля $R=G F(2)=\{0,1\}$ характер $\chi$ задается равенствами $\chi(0)=1$, $\chi(1)=-1$, и в этом случае

$$
C_{u, v}(j, l)=\sum_{i=0}^{l-1}(-1)^{u(i)-v(i+j)} .
$$

Таким образом коэффициент $C_{u, v}(j, l)$ равен разности числа совпадающих координат в векторах $(u(0), \ldots, u(l-1)),(v(j), \ldots, v(j+l-1))$ и числа несовпадающих координат, т. е. он равен разности $l$ и удвоенного расстояния Хемминга между рассматриваемыми векторами. Чем меньше величина $\left|C_{u, v}(j, l)\right|$, тем в большем количестве координат отличаются изучаемые отрезки последовательностей $u$ и $v$. Формула (14) является обобщением формулы (15) на случай произвольного кольца Галуа.

Теорема 6. Пусть $F(x)$ - реверсивный многочлен Галуа степени m над кольиом $R, T(F)=p^{\nu}\left(q^{m}-1\right) / d, u, v-\pi Р \Pi$ надкольиом $R c$ характеристическим многочленом $F(x)$. Тогда для любых таких цельх неотрицательных $j$, что $u \neq x^{j} v, u$ каждого такого натурального числа $l$, что $l<T(\bar{F})$,

$$
\left|C_{u, v}(j, l)\right| \leq\left(\frac{3 p^{\nu} l}{d}\left(q^{m}-l+(l-1)\left(d p^{n-1}-1\right) q^{\frac{m}{2}}\right)\right)^{\frac{1}{3}}
$$

Кроме того, при $n=1$ верна также оченка

$$
\left|C_{u, v}(j, l)\right| \leq\left(3\left(q^{m} l-l^{2}\right)\right)^{\frac{1}{3}}
$$

Доказательство. Пусть $\omega-$ последовательность над кольцом $R$ с элементами $\omega(i)=u(i)-v(i+j), i \geq 0$. По условию $\omega-$ ненулевая ЛРП с характеристическим многочленом $F(x)$. Согласно равенствам $(2)$ и $(14)$ для всех $z \in R$ и $H=\{e\}$

$$
\left|C_{u, v}(j, l)\right|=\left|\sum_{i=0}^{l-1} \chi(\omega(i))\right|=\left|\tau_{l}(z, \omega, H)\right| .
$$

Для завершения доказательства остается воспользоваться теоремами 2 и 4.

Заметим, что для коэффициента кросс-корреляции, определенного формулой (14), справедлива тривиальная оценка: $\left|C_{u, v}(j, l)\right| \leq l$. Будем называть верхнюю оценку величины $\left|C_{u, v}(j, l)\right|$ нетривиальной, если она меньше $l$. Оценка (17) нетривиальна при $l \geq \sqrt{3} q^{m / 2}$, а оценка (16) в частном случае $d=1$ нетривиальна для всех таких $l$, что $l \geq 3 p^{\nu+n-1} q^{m / 2}$. 
Ранее для случая $R=G F(q)$, т. е. при $n=1$, были получены абсолютные оценки коэффициента кросс-корреляции, которые в условиях теоремы 6 имеют вид

$$
\left|C_{u, v}(j, l)\right| \leq q^{\frac{m}{2}}(a \ln T(F)+b),
$$

где $a=1, b=1$ (см. [1], [13, стр. 73], [3, теорема 1]); $a=2 / \pi, b=7 / 5$ (см. [4, следствие 4]); $a=2 / \pi, b=\ln (4 / \pi)$ (см. [5, стр. 686]). Оценка (17) точнее оценки (18) при всех таких $l$, что

$$
l \leq \frac{1}{3} q^{\frac{m}{2}}(a \ln T(F)+b)^{3} .
$$

В [18, теорема 1] для случая $R=\mathbb{Z}_{p^{n}}=G R\left(p^{n}, p^{n}\right), \nu=0, d=1$ была доказана следующая абсолютная оценка:

$$
\left|C_{u, v}(j, l)\right|<p^{\frac{m}{2}+n-1}(m \ln p+1) .
$$

Оценка (16) уточняет это неравенство при всех таких $l$, что

$$
p^{\frac{m}{2}} \leq l \leq \frac{1}{\sqrt{3}} p^{\frac{m}{2}+n-1}(m \ln p+1)^{\frac{3}{2}} .
$$

\section{5. Распределение элементов из заданного подмноже- ства}

Пусть $R=G R\left(q^{n}, p^{n}\right), M$ - некоторое непустое подмножество кольца $R, u$ - последовательность элементов кольца $R$. Рассмотрим вопрос о числе $N_{l}(M, u)$ таких чисел $i \in\{0,1, \ldots, l-1\}$, при которых $u(i) \in M$. Другими словами, число $N_{l}(M, u)$ равно количеству появлений элементов из множества $M$ среди элементов $u(0), u(1), \ldots, u(l-1)$.

Пусть $\chi$ - аддитивный характер кольца $R$, определенный равенством (13). Зададим следующий параметр:

$$
\varkappa(M)=\max _{c \in R \backslash\{0\}}\left|\sum_{b \in M} \chi(c b)\right| .
$$

Обозначим через $C_{l}(F, n)$ правую часть неравенства (16) при $n>1$ и правую часть неравенства (17) при $n=1$.

Теорема 7. Пусть $F(x)$ - реверсивный многочлен Галуа степени $m$ над кольиом $R$. Тогда для каждой ЛРП $и \in L_{R}(F) c\|u\|=0$ и каждого натурального числа $l$, удовлетворяющего условию $l<T(\bar{F})$,

$$
\left|N_{l}(M, u)-\right| M\left|\frac{l}{q^{n}}\right| \leq \frac{q^{n}-1}{q^{n}} \varkappa(M) C_{l}(F, n) .
$$


Доказательство. С использованием соотношений

$$
\sum_{b \in M} \sum_{c \in R} \chi(c(u(i)-b))= \begin{cases}0, & \text { если } u(i) \notin M \\ q^{n}, & \text { если } u(i) \in M\end{cases}
$$

получим

$$
N_{l}(M, u)=\frac{1}{q^{n}} \sum_{i=0}^{l-1} \sum_{b \in M} \sum_{c \in R} \chi(c(u(i)-b)),
$$

а значит,

$$
N_{l}(M, u)-|M| \frac{l}{q^{n}}=\frac{1}{q^{n}} \sum_{c \in R \backslash\{0\}} \sum_{b \in M} \chi(-c b) \sum_{i=0}^{l-1} \chi(c u(i)) .
$$

Так как $\|c u\|=\|c\| \neq n, \chi(-c b)=\overline{\chi(c b)}$ для всех $c \in R \backslash\{0\}, b \in M$, то, переходя в равенстве $(21)$ к исследованию абсолютных величин и используя теоремы 2 и 4, получим

$$
\begin{aligned}
\left|N_{l}(M, u)-\right| M\left|\frac{l}{q^{n}}\right| & \leq \frac{q^{n}-1}{q^{n}} \max _{c \in R \backslash\{0\}}\left|\sum_{b \in M} \chi(-c b)\right|\left|\sum_{i=0}^{l-1} \chi(c u(i))\right| \leq \\
& \leq \frac{q^{n}-1}{q^{n}} \varkappa(M) C_{l}(F, n) .
\end{aligned}
$$

Заметим, что величина $|M| l / q^{n}$ является "естественным" средним значением для числа появлений элементов из множества $M$ на отрезке длины $l$ в последовательности $u$.

Имеет место тривиальная оценка $\varkappa(M) \leq|M|$. В общем случае эту оценку не удается уточнить. Пусть $R=P=G F(q)$. Рассмотрим некоторые подмножества $M \subset P$ для которых это удается сделать.

Следствие 1. Пусть $R=P$ в условиях теоремы 7, тогда

1) если $M$ - множество всех элементов вида $a^{k}$, где $a \in P, k-$ делитель $q-1$, mо

$$
\left|N_{l}(M, u)-\frac{(q+k-1) l}{k q}\right| \leq \frac{(q-1)(k-1)}{q k}(\sqrt{q}+1)\left(3\left(q^{m} l-l^{2}\right)\right)^{\frac{1}{3}},
$$

2) если $M^{\prime}=P \backslash M$, где $M$ определено в $n$. 1), то

$$
\left|N_{l}\left(M^{\prime}, u\right)-\frac{(k-1)(q-1) l}{k q}\right| \leq \frac{(q-1)(k-1)}{q k}(\sqrt{q}+1)\left(3\left(q^{m} l-l^{2}\right)\right)^{\frac{1}{3}},
$$


3) если $M-$ подгруппа группы $P^{*}$, имеющая порядок $(q-1) / k$, то

$$
\left|N_{l}(M, u)-\frac{(q-1) l}{k q}\right| \leq \frac{(q-1)((k-1) \sqrt{q}+1)}{q k}\left(3\left(q^{m} l-l^{2}\right)\right)^{\frac{1}{3}} .
$$

Доказательство. 1) Пусть $c$ - произвольный ненулевой элемент поля $P$, а $\gamma$ - примитивный элемент поля $P$, тогда

$$
\sum_{b \in M} \chi(c b)=1+\sum_{i=0}^{((q-1) / k)-1} \chi\left(c \gamma^{k i}\right)=1+\frac{1}{k} \sum_{i=0}^{q-2} \chi\left(c\left(\gamma^{i}\right)^{k}\right)=\frac{k-1}{k}+\frac{1}{k} \sum_{x \in P} \chi\left(c x^{k}\right)
$$

Переходя к исследованию абсолютных величин, с использованием оценки для сумм Вейля (см., например, [2, теорема 5.32]), получим

$$
\left|\sum_{b \in M} \chi(c b)\right| \leq \frac{1}{k}\left|\sum_{x \in P} \chi\left(c x^{k}\right)\right|+\frac{k-1}{k} \leq \frac{(k-1) \sqrt{q}}{k}+\frac{k-1}{k}=\frac{k-1}{k}(\sqrt{q}+1) .
$$

2) Оценка непосредственно следует из п. 1) и равенства $N_{l}\left(M^{\prime}, u\right)=l-$ $N_{l}(M, u)$.

3) В этом случае

$$
\sum_{b \in M} \chi(c b)=\frac{1}{k} \sum_{x \in P^{*}} \chi\left(c x^{k}\right)=\frac{1}{k} \sum_{x \in P} \chi\left(c x^{k}\right)-\frac{1}{k}
$$

и, следовательно,

$$
\varkappa(M) \leq \frac{1}{k}+\frac{1}{k} \max _{c \in P^{*}}\left|\sum_{x \in P} \chi\left(c x^{k}\right)\right| \leq \frac{1}{k}+\frac{k-1}{k} \sqrt{q}=\frac{(k-1) \sqrt{q}+1}{k} .
$$

Отметим, что в [1] и [19] элементы множества $M$, рассмотренного в п. 3) следствия 1 , названы вычетами степени $k$, а элементы множества $M^{\prime}$, рассмотренного в п. 2), названы невычетами степени $k$ (при этом 0 не относится ни к одному из этих классов). В этой терминологии множество $M$, рассмотренное в п. 1$)$, состоит из всех вычетов степени $k$ и нулевого элемента. В [1, теорема 2] и [19, стр. 202] получены оценки, аналогичные оценкам из следствия 1 , которые отличаются лишь заменой величины $\left(3\left(q^{m} l-l^{2}\right)\right)^{\frac{1}{3}}$ множителем $q^{\frac{m}{2}}(\ln T(F)+1)$. Как было отмечено в предыдущем параграфе, оценки из следствия 1 точнее при всех таких $l$, что

$$
l \leq \frac{1}{3}(\ln T(F)+1)^{3} q^{\frac{m}{2}} .
$$


В [20, теорема 4] (см. также [21]) было показано, что в оценках из работ [1] и [19] величину $q^{m / 2}$ можно заменить на $q^{(m-1) / 2}$, при этом сами оценки выписаны с точностью до главного множителя при $q \rightarrow \infty$, что не позволяет провести их сравнение с оценками из следствия 1. Отметим лишь, что если параметры $q$ и $k$ зафиксированы, а $m \rightarrow \infty$, то для ЛРП $u$ максимального периода $q^{m}-1$ оценки из следствия 1 будут точнее рассматриваемых абсолютных оценок при $l=O\left(m^{3} q^{m / 2}\right)$.

Обозначим через $D_{l}(F, n, k)$ правую часть неравенства из теоремы 5 при $n>1$ и правую часть неравенства из теоремы 3 при $n=1$.

Теорема 8. Пусть выполнены условия теоремы $7, T(\bar{F})=t u$

$$
l<\frac{t q}{2(q-1)}, \quad l \leq \frac{t}{(t, q-1)},
$$

тогда справедлива оченка

$$
\left|N_{l}(M, u)-\right| M\left|\frac{l}{q^{n}}\right| \leq \frac{|M|}{q^{n}} \sum_{k=0}^{n-1} \frac{D_{l}(F, n, k)}{q^{k}} .
$$

Доказательство. Согласно равенствам (2) и (21)

$$
\begin{aligned}
N_{l}(M, u)-|M| \frac{l}{q} & =\frac{1}{q^{n} f} \sum_{a \in R^{*}} \sum_{c \in R \backslash\{0\}} \sum_{b \in M} \chi(-a c b) \sum_{i=0}^{l-1} \chi(a c u(i))= \\
& =\frac{1}{q^{n} f} \sum_{b \in M} \sum_{c \in R \backslash\{0\}} \tau_{l}(c b, c u, H),
\end{aligned}
$$

где $H=R^{*}, f=\left|R^{*}\right|=q^{n-1}(q-1)$. Отсюда

$$
N_{l}(M, u)-|M| \frac{l}{q}=\frac{1}{q^{n} f} \sum_{b \in M} \sum_{k=0}^{n-1} \sum_{c \in R,\|c\|=k} \tau_{l}(c b, c u, H) .
$$

Так как $\|u\|=0$, то $\|c u\|=\|c\| \leq\|c b\|$ для всех $c \in R \backslash\{0\}, b \in M$. Число таких элементов $c \in R$, что $\|c\|=k$, где $k<n$, равно $(q-1) q^{n-k-1}$, поэтому с использованием теорем 3 и 5 получим

$$
\left|N_{l}(M, u)-\right| M\left|\frac{l}{q^{n}}\right| \leq \frac{1}{q^{n} f} \sum_{b \in M} \sum_{k=0}^{n-1}(q-1) q^{n-k-1} D_{l}(F, n, k)=
$$




$$
=\frac{|M|}{q^{n}} \sum_{k=0}^{n-1} \frac{D_{l}(F, n, k)}{q^{k}} .
$$

Сравним оценки из теорем 7 и 8. Будем предполагать, для удобства сравнения, что величина $\varkappa(M)$ равна $|M|$. Такое предположение выполнено, например, для каждого одноэлементного множества $M$. Пусть $n=1(R=G F(q))$, тогда при $q=2$ оценки совпадают, а при $q \geq 3$ оценка из теоремы 8 является точнее для всех таких $l$, что $l<q^{m} / 2$. В ситуации, когда $n>1$, a $d=1$, несложно показать, что оценка из теоремы 8 является точнее при всех $l$ таких, что $l<\left(q^{m}-\left(p^{n-1}-1\right) q^{m / 2}\right) / 2$.

\section{Список литературы}

[1] Коробов Н.М. Распределение невычетов и первообразных корней в рекуррентных рядах // Доклады АН СССР. - 1953. - Т. 88. № 4. - C. 603-606.

[2] Лидл Р., Нидеррайтер Г. Конечные поля. - М. : Мир, 1988. - 822 с.

[3] Нечаев В.И. Распределение знаков в последовательности прямоугольных матриц над конечным полем // Труды МИАН. - 1997. - T. 218. - C. 335-342.

[4] Niederreiter H. Distribution properties of feedback shift register sequences // Probl. Control and Inform. Theory. - 1986. - V. 15. № 1. - P. 19-34.

[5] Sarwate D. V. An upper bound on the aperiodic autocorrelation function for a maximal-length sequence // IEEE Trans. Inf. Theory. - 1984. V. 30. № 4. - P. 685-687.

[6] Сидельников В.М. Оценки для числа появлений знаков на отрезках рекуррентной последовательности над конечным полем // Дискрет. матем. - 1991. - Т. 3. № 2. - С. 87-95.

[7] Niederreiter H., Shparlinski I.E. On the distribution of inversive congruential pseudorandom numbers in path of the period // Math. Comput. - 2001. - V. 70. № 236. - P. 1569-1574.

[8] Niederreiter H., Winterhof A. Multiplicative character sums for nonlinear recurring sequences // Acta Arith. - 2004. - V. 113. № 3. P. 299-305. 
[9] Burde K. Verteilungseigenschaften von potenzresten // J. Reine und Angew. Math. - 1971. - Band 249. - P. 133-172.

[10] Камловский О.В. Оценки частот появления нулей в линейных рекуррентных последовательностях векторов // Чебышевский сборник. 2005. - Т. 6. Вып. 1. - С. 135-146.

[11] Камловский О.В. Метод В.М. Сидельникова для оценки числа знаков на отрезках линейных рекуррентных последовательностей над кольцами Галуа // Матем. заметки. - 2012. - Т. 91. Вып. 3. - С. $371-382$.

[12] Камловский О.В. Уточнение оценок для числа появлений элементов в линейных рекуррентных последовательностях над кольцами Галуа // Фунд. и прикл. матем. - 2012. - Т. 17. Вып. 7. - С. 97-115.

[13] Коробов Н.М. Тригонометрические суммы и их приложения. - М. : Наука, 1989. - 240 с.

[14] Биляк И.Б. Оценки числа появлений элементов на отрезках линейных рекуррентных последовательностей // Прикл. дискрет. матем. - 2013. - № 1(19). - C. 5-13.

[15] Нечаев А.А. Код Кердока в циклической форме. Дискрет. матем. 1989. - T. 1. № 4. - С. 123-139.

[16] Нечаев A.А. Цикловые типы линейных подстановок над конечными коммутативными кольцами // Матем. сб. - 1993. - Т. 184. № 3. C. $21-56$.

[17] Kumar P.V., Helleseth T., Calderbank A.R. An upper bound for Weil exponential sums over Galois rings and applications // IEEE Trans. Inform. Theory. - 1995. - V. 41. № 2. - P. 456-468.

[18] Shanbhag A.G., Kumar P.V., Helleseth T. Upper bound for a hybrid sum over Galois rings with applications to aperiodic correlation of some $q$-ary sequences // IEEE Trans. Inform. Theory. - 1996. - V. 42. № 1 . - P. 250-254.

[19] Нечаев В.И., Степанова Л.Л. Распределение невычетов и первообразных корней в рекуррентных последовательностях над полем алгебраических чисел // Успехи матем. наук. - 1965. - Т. 20. № 3. С. $197-203$. 
[20] Шпарлинский И.Е. Распределение невычетов и первообразных корней в рекуррентных последовательностях // Матем заметки. - 1978. - T. 24. № 5. - C. 603-613.

[21] Шпарлинский И.Е. О распределении значений рекуррентных последовательностей / / Проблемы передачи информации. - 1989. - Т. 25. № 2. - C. $46-53$. 